\title{
New Kalman Filter Residue-Based Identification and Soft Sensor Design for Accurate Trajectory Tracking with a Fault-tolerant Robot
}

\author{
Rajamani Doraiswami ${ }^{1}$, Lahouari Cheded ${ }^{2}$ and $\{$ Eduardo Jair Tito Mamani, Pamela Giselle \\ Villarroel, Paul Gerardo Cori Mamani, Paulo Roberto Loma Marconi, Claudio Cesar Carlos Olivares, \\ Justo Franz Choque Choque, Layde Aydee Cruz Torrico, Layde Aydee Cruz Torrico ${ }^{3}$ \\ ${ }^{1}$ Department of Electrical and Computer Engineering, University of New Brunswick, Fredericton \\ New Brunswick, Canada (dorai@unb.ca) \\ ${ }^{2}$ Professor. Independent Researcher and Consultant, Life Senior IEEE Member. UK (cheded@kfupm.edu.sa) \\ ${ }^{3}$ Universidad Mayor de San Andrés (UMSA), La Paz - Bolivia. (paulo.loma@iee.org)
}

\begin{abstract}
A Kalman filter(KF)-based identification, internal model-based controller for accurate tracking a specified trajectory despite the sensor errors, and fault tolerance is proposed. This study was mainly motivated by the need for precision, resolution and accuracy required in robotic applications such as robotic surgery. The computed torque approach is used to map a nonlinear model into a linear one. The sensor errors of the orientation input and the position corrupted by unknown input and output stochastic disturbance and measurement noise. Predictive analytics is used to estimate the true input by exploiting its smoothness and the randomness of the noisy input. The system is described using the Box-Jenkins(BJ) model, which is an augmented model of the true output, termed signal and the disturbance. The BJ model and the associated KF are identified without the a priori knowledge of the statistics of the disturbance and measurement noise. Using the key properties of KF the signal, the output error, the signal model, and the disturbance models, the KF associated with the signal model is accurately identified. An internal model-based state-feedback and feedforward controller is designed to accurately track the desired trajectory. The hardware sensors are replaced by KF-based sensors. The KF ensures fault tolerance. The proposed scheme was successfully evaluated on a physical robot.
\end{abstract}

Keywords: robotic surgery, Euler Lagrange Equation, identification, Box-Jenkins model, Kalman filter, soft sensor.

\section{Introduction}

The prime motivation for this study is to achieve accurate tracking of the desired robot arm trajectory in the face of unknown disturbances and measurement noises [1-5]. This is vital in, for example, robotic surgery, of either the non-invasive or the minimally-invasive type, where delicate operations, requiring the utmost accuracy, are carried out on vital organs, such as eyes, heart, brain, spinal cord, throat and knees [3].

\subsection{Sensors for Input and Output Measurement}

The actuators at the joint angles control the position of the end effectors. The orientation is made of the joint angles, namely the roll $\phi$, pitch $\theta$ and yaw $\psi: \boldsymbol{\eta}=(\phi, \theta, \psi) \in R^{3}$, that are termed as input, and the coordinate axes $\xi=(x, y, z) \in R^{3}$ are the position of the end-effector. Using the inverse kinematics, the input values are selected so that the output tracks a specified trajectory. To ensure accurate tracking, hardware sensors are used to measure both the input and output and they include: (a) Accelerometer to measure the inertial acceleration, (b) Gyroscope to measure angular velocity about the defined axis, and (c) Magnetometer to be used along with the gyroscope to get better estimates of the robot's orientation.

The hardware sensors are required to ensure precision, resolution and accuracy, but they are maintenance-prone and are affected by disturbances and measurement noise. Thus, these are replaced by accurate, reliable, and consistent KF-based soft sensor, which are proposed here.

\subsection{Predictive Analytics}

Predictive analytics is of paramount importance in resolving a host of problems in a variety of fields including engineering, financial and medical and relies on a wide range of tools such as statistical modelling and analytical techniques, machine learning, artificial intelligence, and deep learning algorithms [6-8]. Here, the true input is estimated from the 
corrupted input by exploiting the smoothness of the true input and the randomness of the corrupted input using two-stage frequency domain identification [8]. It is assumed that dominant peaks in the spectrum of the corrupted input are associated with the true input and the model that generates true input such as constants or sinusoids has a rational transfer function with poles and zeros. The resulting system model relates true input and the true output corrupted by disturbance and measurement noise. For notational convenience, estimated true input is termed merely as input.

\subsection{Dynamic Model Of A Robot}

A dynamic model of the robot relating the estimated true input and the output is derived using the Euler-Lagrange equation. The states of the system are the joint variables, their first and second derivatives. The model of the robot is an unstable second-order nonlinear one that relates the second-order derivatives of the joint angles to the nonlinear forces and torques including the centripetal/Coriolis force, gravitational force, and the viscous friction.

\subsubsection{Feedback Linearization Approach}

One of the most common approaches to the design of robot controllers is a computed-torque controller, also known as the inverse dynamics controller. It is based on the feedback linearization principle which is an approach that maps a nonlinear model into a linear one and treats it as such [5]. An exact linear model is obtained by introducing new variables termed control inputs to replace all nonlinear terms including the applied forces, applied torques, Coriolis forces, and gravitational load.

\subsubsection{System Model: Box-Jenkins Model}

An augmented model of the system, termed Box-Jenkins model formed of a) the signal model relates the estimated input and disturbance and measurement noise-free output, b) the model of the stochastic disturbance driven by random disturbance input. The output is the sum of the signal, the stochastic disturbance and measurement noise.

\subsubsection{Kalman Filter and Its Key Properties}

The key properties of the KF are exploited to estimate the signal from the output corrupted by disturbance and measurement noise and obtain derivative-free estimates of the joint. The following key properties are restated here for convenience [15-17]:

P1: The residual of the Kalman filter is a zero-mean white noise process if and only if there is no mismatch between the actual model of the system and its identified model embodied in the Kalman filter, and its variance is minimum.

P2: The estimate is optimal in the sense that it is the best estimate that can be obtained by any estimator in the class of all estimators that are constrained by the same assumptions.

P3: The signal is extracted from the output corrupted by stochastic disturbance and measurement noise. The signal model is derived from the residual model of the KF.

\subsection{Identification of the Robot Model}

The KF associated with the system plays a crucial role in the system identification, fault detection and isolation, and estimation of the signal, the output error (sum of the disturbance and measurement noise), signal and disturbance model in view of its following key properties:

- The above-stated property P1. If there is a model mismatch, then the residual will not be a zero-mean white noise process and an additive term termed fault-indicative term. The fault-indicative term is a filtered version of the deviation in the linear regression model of the system or that of the signal. The fault indication term is employed in fault detection and fault isolation

- If the identified nominal system model is accurate, then as per property P1, the following estimates derived using the $\mathrm{KF}$ are accurate in the sense that they are true and free from the disturbance and measurement noise, unlike the system output:

- Estimates of the signal and its model

- Estimates of the output error and disturbance model 
- The KF for the signal model relating the estimated signal and the input is accurately identified.

The static and dynamic behaviors of the physical systems change as a result of variations of the parameters of the subsystems such as sensors, actuators, pumps, and disturbance models. As the parameters of the subsystems are not generally accessible, novel emulators are used to mimic these model perturbations. An emulator takes the form of a static gain or an all-pass filter to induce gain or phase variations in the subsystem it is connect to. Emulator parameters are perturbed to mimic various normal and abnormal, or faulty, operating scenarios resulting from variations in these subsystems. Emulators are connected to the accessible inputs and outputs in cascade with the subsystems such as the actuator and sensor comprising the signal model, and disturbance models during the offline system identification. The emulator-generated data covers both normal and abnormal operating scenarios including various types of faults. The emulator-generated data is employed in a) the identification of the system, the KF-based identification, and fault isolation. The fault-free or nominal system and the associated KF are identified using the emulator-generated data. In system identification, the criterion for determining whether the identified model has captured completely the static and the dynamic behavior of the system is that the residual of the KF associated with the system is a zero-mean white noise process. If the equation error is a zero-mean white noise process, the estimate will be unbiased and efficient; otherwise, it will be biased. In view of the above-stated property, the residual then becomes a zero-mean white noise process if and only if the system and the identified model embodied in the KF are identical. The structure of the residual model of the KF, and not that of the linear regression model of the system, is employed in identification. The identification objective of ensuring the residual (innovation sequence) is a zero-mean white noise process, will ensure not only that the KF is accurately identified but also that the Kalman gain is optimal, thereby avoiding the need to specify the covariance of the disturbance and the measurement noise and to use the Riccati equation to solve for the Kalman gain.

\subsection{Kalman Filter-Based Feedback-Feedforward Controller}

In the literature, many approaches to the design of a controller for tracking such as proportional (P), proportional-integral and derivative (PID), adaptive, and nonlinear [9-14]. A KF-based combined feedback and feed forward controller is designed here using the internal principle for the the feedback controller [18]:

- An internal model of the reference is included in the controller. Recall that an internal model of a signal is a transfer matrix whose zero-input response is copy of the signal.

- The internal model is driven by the tracking error, which is error between the reference trajectory and the estimate of the signal, rather than the estimate of the output, which is the signal corrupted by the disturbance and measurement noise

- The closed-loop system is asymptotically stable despite model perturbations

The feedforward controller rejects quickly the disturbance without waiting for the deviation in the output to occur. Thanks to its predictive nature, this controller is pro-active. It overcomes sluggish dynamics and delays without compromising the stability of the system.

\subsection{Derivative-free soft sensor}

The KF-based soft sensor is employed to estimate accurately the joint variables and their first and second derivarives despites the srochastic disturbance and measurement noise. The proposed soft sensor is an extension of the scheme proposed in [19] to the output measurements corrupted by disturbances and the measurement noise. Thanks to the availability of accurate estimation of the joint variables and their derivatives, nonlinear terms, which are functions of joint variables and their derivatives, can be computed and the computer torque approach can be used to map the nonlinear system to the linear one.

\subsection{Main Contributions:}

The main contribution is the development of the combined state-feedback and feedforward controller to track accurately the desired trajectory despite deterministic and unknown stochastic disturbance and measurement noise. Other specific contributions include the following: 
a) An internal model-based state-feedback and feedforward controller to track the desired trajectory despite the deterministic disturbance is developed, and the closed loop system is derived

b) Using predictive analytics, the true input is extracted from the corrupted input

c) The Kalman filters for the system and the signal models of the resulting closed-loop system obtained in a) is identified without the use of any a-priori knowledge or use of the statistics of the disturbance and measurement noise. Using the above KF's key properties, the signal and the states of the signal model are estimated.

d) Finally, the KF-based controller to track accurately the desired trajectory despite the stochastic disturbance and measurement noise is obtained

The proposed autonomous control scheme was evaluated successfully on a physical robot constructed by the graduate students of the Universidad Mayor de San Andres, La Paz Bolivia.

\section{Kinematics and Inverse Kinematics}

2.1 Homogeneous Transformation

We will develop the kinematic model by sequentially starting from the first joint, then the second one, and so on till the last one where the present joint $i$ and the previous one $(i-1)$ are denoted by joint variables $\boldsymbol{q}_{i}=\left(x_{i}, y_{i}, z_{i}\right) \in R^{3}$ and $\boldsymbol{q}_{i-1}=\left(x_{i-1}, y_{i-1}, z_{i-1}\right) \in R^{3}$ where $\boldsymbol{q}_{i}$ is defined with respect to the $i^{\text {th }}$ Cartesian coordinate space, and the previous joint variables $\boldsymbol{q}_{i-1}$, which is defined with respect to the $(i-1)^{t h}$ Cartesian coordinates. The joint variables are $\boldsymbol{q}_{i}$ obtained from subjecting $\boldsymbol{q}_{i-1}$ to rotation and translation $\boldsymbol{d}_{i-1}^{i} \in R^{3}$.

$$
\boldsymbol{q}_{i}=\boldsymbol{R}_{i-1}^{i} \boldsymbol{q}_{-1}+\boldsymbol{d}_{i-1}^{i}
$$

Where $\boldsymbol{R}_{i-1}^{i} \in S O(3)$ is the orthonormal rotational transformation; and $\boldsymbol{d}_{i-1}^{i} \in R^{3}$ is the distance between the origins along the $x$-axis, $y$-axis and $z$-axis. At the joint $i$ the Denavit-Hartenberg (DH) transformation $\boldsymbol{A}_{i-1}^{i} \in R^{4 \times 4}$, which is invertible, relates the joint variables $\boldsymbol{q}_{i}$, and the previous joint variables $\boldsymbol{q}_{i-1}$ by:

$$
\boldsymbol{A}_{i-1}^{i}=\left[\begin{array}{cc}
\boldsymbol{R}_{i-1}^{i} & \boldsymbol{d}_{i-1}^{i} \\
0 & 1
\end{array}\right] ;\left(\boldsymbol{A}_{i-1}^{i}\right)^{-1}=\left[\begin{array}{cc}
\left(\boldsymbol{R}_{i-1}^{i}\right)^{T} & -\left(\boldsymbol{R}_{i-1}^{i}\right)^{T} \boldsymbol{d}_{i-1}^{i} \\
0 & 1
\end{array}\right]
$$

The DH transformation relating $\boldsymbol{q}_{i}$ and $\boldsymbol{q}_{i-1}$ is:

$$
\left[\begin{array}{c}
\boldsymbol{q}_{i} \\
1
\end{array}\right]=\boldsymbol{A}_{i-1}^{i}\left[\begin{array}{c}
\boldsymbol{q}_{i-1} \\
1
\end{array}\right] ;\left[\begin{array}{c}
\boldsymbol{q}_{i-1} \\
1
\end{array}\right]=\left(\boldsymbol{A}_{i-1}^{i}\right)^{-1}\left[\begin{array}{c}
\boldsymbol{q}_{i} \\
1
\end{array}\right]
$$

\subsection{The inverse kinematics}

Let $\boldsymbol{p}_{d}=\left(x_{d}, y_{d}, z_{d}\right) \in R^{3}$ denote the position of the end-effector in Euclidian space and is described with respect to a reference frame with coordinates axes denoted $x, y$, and $z$. The function $\varphi($.$) relating \boldsymbol{p}_{d}$ and the joint angles $\boldsymbol{q}_{d}=\left(\theta_{1 d}, \theta_{2 d}, \theta_{3 d}\right) \in R^{3}$ controlled by the actuators of the robot are nonlinear and are given by:

$$
\boldsymbol{p}_{d}=\varphi\left(\boldsymbol{q}_{d}\right)
$$

In order to meet this requirement, the joint angle $\boldsymbol{p}_{d}$ has to be determined for a given desired trajectory $\boldsymbol{p}_{d}$. Hence the inverse kinematics is posed as an optimization problem:

$$
\boldsymbol{q}_{d}^{*}=\min _{\boldsymbol{q}_{d}}\left\|\boldsymbol{p}_{d}-\varphi\left(\boldsymbol{q}_{d}\right)\right\|^{2}
$$

The optimal solution $\boldsymbol{q}_{d}^{*}$ is employed and the desired motions of the end effector remain within the workspace. 


\section{Dynamic Model of a Robot Manipulator}

The dynamic model is generally derived using the Euler-Lagrange formulation, based on the derivation of the kinetic and the potential energy. A robot is in general formed of links and combination prismatic and revolute joints. A robot with 6 degrees of freedom, 3 for position and 3 for orientation, and $n$ joints which are described by state variables formed of generalized position $\boldsymbol{q}=\left[\begin{array}{llll}q_{1} & q_{2} & \cdot & q_{n}\end{array}\right]^{T} \in R^{n}$, velocity $\dot{\boldsymbol{q}}=\left[\begin{array}{llll}\dot{q}_{1} & \dot{q}_{2} & \cdot \dot{q}_{n}\end{array}\right]^{T} \in R^{n}$ and the acceleration $\ddot{\boldsymbol{q}}=\left[\begin{array}{llll}\ddot{q}_{1} & \ddot{q}_{2} & \cdot \ddot{q}_{n}\end{array}\right]^{T} \in R^{n}$ , and the generalized toque/force $\tau=\left[\begin{array}{llll}\tau_{1} & \tau_{2} & \cdot \tau_{n}\end{array}\right]^{T} \in R^{n}$. The dynamic model takes the following general form [1-5]:

$$
\boldsymbol{M}(\boldsymbol{q}) \ddot{\boldsymbol{q}}+N(\boldsymbol{q}, \dot{\boldsymbol{q}})=\tau
$$

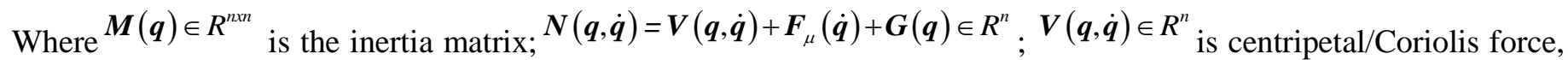
$\boldsymbol{G}(\boldsymbol{q}) \in R^{n}$ gravitational force, the viscous friction $\boldsymbol{F}_{\mu}(\dot{\boldsymbol{q}}) \in R^{n}$ and $\tau$ is the generalized force/torque input that controls the joint torques in each actuator. It is assumed that $\boldsymbol{M}(\boldsymbol{q})$ is an invertible positive definite matrix. Multiplying both sides of (6) by $\boldsymbol{M}^{-1}(\boldsymbol{q})$ yields:

$$
\ddot{\boldsymbol{q}}=\boldsymbol{M}^{-1}(\boldsymbol{q})(\tau-N(\boldsymbol{q}, \dot{\boldsymbol{q}}))
$$

\subsection{Computed-Torque Controller}

The computed torque approach is widely used in autonomous unmanned systems such as robots and drones, and is based on the feedback linearization principle which is an approach that maps a nonlinear model into a linear one [1], [5-6]. The control objective is that the end effector tracks a desired trajectory. $\boldsymbol{p}_{d}=\left(x_{d}, y_{d}, z_{d}\right) \in R^{3}$.

A new variable termed control input $\boldsymbol{u}=\left[\begin{array}{llll}u_{1} & u_{2} & \cdot & u_{n}\end{array}\right]^{T} \in R^{n}$ is introduced with a view to eliminating the nonlinear

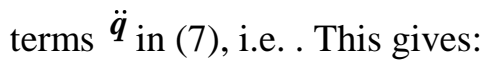

$$
\boldsymbol{u}=\boldsymbol{M}^{-1}(\boldsymbol{q})(\tau-\boldsymbol{N}(\boldsymbol{q}, \dot{\boldsymbol{q}}))
$$

The expression for the torque input $\tau$ derived using (8):

$$
\tau=\boldsymbol{M}(\boldsymbol{q}) \boldsymbol{u}+\boldsymbol{N}(\boldsymbol{q}, \dot{\boldsymbol{q}})
$$

The torque input $\tau$ is applied to physical robot model (8)

Substituting $\boldsymbol{u}$ yields Error! Reference source not found. becomes:

$$
\ddot{\boldsymbol{q}}(t)=\boldsymbol{u}(t)
$$

\subsection{Box-Jenkins model}

The Box-Jenkins model covers several imporatnt practical systems, including for example, autonomous vehicles such as drones and aerial vehicles. The Auto-Regressive and Moving Average (ARMA), Moving Average (MA), and Auto Regressive (AR) models are all special cases of the Box-Jenkins model.

\subsubsection{Signal model}

The discrete-time state space model $\left(\boldsymbol{A}_{p}, \boldsymbol{B}_{p}, \boldsymbol{C}_{p}\right)$ of (10) is:

$$
\begin{aligned}
& \boldsymbol{x}_{p i}(k+1)=\boldsymbol{A}_{p} \boldsymbol{x}_{p i}(k)+\boldsymbol{B}_{p} u_{i}(k) \\
& q_{i}(k)=\boldsymbol{C}_{p} \boldsymbol{x}_{p i}(k)
\end{aligned}
$$

Where $\boldsymbol{x}_{p i}(t) \in R^{n_{p}} ; \boldsymbol{A}_{p i} \in R^{n_{p} x n_{p}} ; \boldsymbol{B}_{p i} \in R^{n_{p} x 1} ; \boldsymbol{C}_{p i} \in R^{1 x n_{p}} ; n_{p i}=2 u_{i} \in R ; q_{i} \in R$. This model $\left(\boldsymbol{A}_{p i}, \boldsymbol{B}_{p i}, \boldsymbol{C}_{p i}\right)$ is termed as signal model, and $q_{i}$ is termed as a signal. The signal model is unstable. 
Stochastic disturbance: The stochastic disturbance is modelled as an output of a linear time-invariant system $\left(\boldsymbol{A}_{d r i}, \boldsymbol{B}_{d r i}, \boldsymbol{C}_{d r i}\right)$ driven by a zero-mean Gaussian white noise process $w_{i}(k)$. The state-space model of the stochastic disturbance is:

$$
\begin{aligned}
& \boldsymbol{x}_{d r i}(k)=\boldsymbol{A}_{d r i} \boldsymbol{x}_{d r i}(k)+\boldsymbol{B}_{d r i} w_{i}(k) \\
& d_{r i}(k)=\boldsymbol{C}_{d r i} \boldsymbol{x}_{d r i}(k)
\end{aligned}
$$

Where $\boldsymbol{x}_{d r i} \in R^{n_{d r}} ; \boldsymbol{A}_{d r} \in R^{n_{d r} x n_{d r}} ; \boldsymbol{B}_{d r} \in R^{n_{d r}} ; \boldsymbol{C}_{d r} \in R^{n n n_{d r}}$.

\subsubsection{Measurement Output}

Let the output measurement is $\boldsymbol{y}(k)=\left[\begin{array}{llll}y_{1}(k) & y_{2}(k) & . & y_{n}(k)\end{array}\right]^{T} \in R^{n}$. The $i^{\text {th }}$ output $y_{i}(k)$ is expressed as a sum of the joint's variable $q_{i}(k)$, the stochastic disturbance $d_{r i}(k)$ and deterministic disturbance, $d_{d d i}(k)$ and the measurement noise $v_{i}(k)$ and is given by:

$$
y_{i}(k)=q_{i}(k)+d_{r i}(k)+v_{i}(k)
$$

The measurement noise is the result of measurement errors in sensors. The output error $\vartheta_{i}(k)$ is defined as a sum of $d_{r i}(k), d_{d d i}(k)$ and $v_{i}(k)$ that corrupts $e_{q i}(k)$, i.e.:

$$
\begin{aligned}
& \vartheta_{i}(k)=y_{i}(k)-q_{i}(k)=d_{r i}(k)+d_{d d i}(k)+v_{i}(k) \\
& \vartheta_{r d i}(k)=d_{r i}(k)+d_{d d i}(k)
\end{aligned}
$$

\subsubsection{Open Loop System: Box-Jenkins Model}

The augmented state-space model $\left(\boldsymbol{A}_{i}, \boldsymbol{B}_{i}, \boldsymbol{C}_{i}\right)$ that generates measurement output, $y_{i}(k) \in R$ is formed of the signal model Error! Reference source not found., the stochastic disturbance model Error! Reference source not found. and the deterministic disturbance model Error! Reference source not found. given by:

$$
\begin{aligned}
& \boldsymbol{x}_{i}(k+1)=\boldsymbol{A}_{i} \boldsymbol{x}_{i}(k)+\boldsymbol{B}_{i} \boldsymbol{r}_{i}(k) \\
& y_{i}(k)=\boldsymbol{C}_{i} \boldsymbol{x}_{i}(k)+v_{i}(k)
\end{aligned}
$$

Where $\boldsymbol{r}_{i}(k)=\left[\begin{array}{c}u_{i}(k) \\ w_{i}(k)\end{array}\right] \in R^{2}, \boldsymbol{C}_{i}=\left[\begin{array}{cc}\boldsymbol{C}_{p i} & \boldsymbol{C}_{d r i}\end{array}\right] \in R^{2 x n_{i}}: \boldsymbol{x}_{i}=\left[\begin{array}{c}\boldsymbol{x}_{p i} \\ \boldsymbol{x}_{d r i}\end{array}\right] \in R^{n_{i}} ; \boldsymbol{A}_{i}=\left[\begin{array}{cc}\boldsymbol{A}_{p i} & 0 \\ 0 & \boldsymbol{A}_{d r i}\end{array}\right] \in R^{n_{i} x n_{i}} ; \boldsymbol{B}_{i}=\left[\begin{array}{cc}\boldsymbol{B}_{p i} & 0 \\ 0 & \boldsymbol{B}_{d r i}\end{array}\right] \in R^{n_{i} x 2}$;

This model, termed as the Box-Jenkins model, is unstable:

Assumptions: (a) It is assumed that the signal ${ }^{q_{i}}$, stochastic disturbance $d_{r i}$ and the measurement noise $v_{i}$ are mutually uncorrelated implying that the inputs $u_{i}$ and $w_{i}$ are uncorrelated too. And (b) The disturbance models $\left(\boldsymbol{A}_{d r i}, \boldsymbol{B}_{d r i}, \boldsymbol{C}_{d r i}\right)$ is

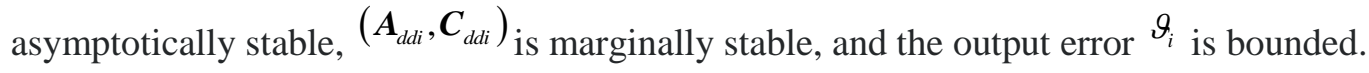

\section{Kalman Filter-Based Controller Design 4.1 Internal Model Principle}

The internal model principle is formulated only for the case when the disturbance is deterministic and there is no measurement noise [12-13]. After identification of the KFs for the system and the signal model, The KF estimates the signal buried in the stochastic disturbance and measurement noise and a novel combined KF and internal model approach is employed.

\subsubsection{Control Objective}

The objective here s to design a state-feedback controller to meet the following objectives:

- The closed-loop system is asymptotically stable 
- The output of the closed-loop system tracks the reference $q_{d i}(k)$ despite the deterministic disturbance $d_{d d i}$ in the absence of a zero-mean stochastic disturbance and measurement noise, that is the expected value of the tracking error is asymptotically zero:

$$
\operatorname{limit}_{k \rightarrow \infty}\left\{E\left[e_{t r i}(k)\right]\right\}=0
$$

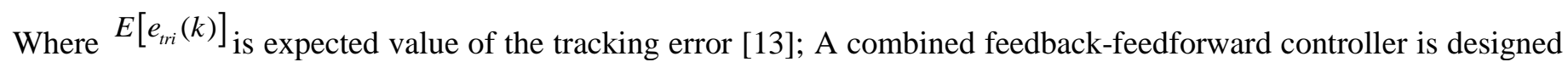
using the internal model principle. The feedback controller ensures tracking of the desired reference despite the deterministic disturbance $d_{d d i}$ and perturbation in the system, while the feedforward controller takes an anticipatory (or predictive) action by injecting the reference in a feedforward manner [6].

The control input $u_{i}(k)$ is generated by the combination of the feedback control $u_{i j b}(k)$ and the feedforward controller output $u_{i f f}(k)$ and is given by:

$$
u_{i}(k)=u_{i f b}(k)+u_{i f f}(k)
$$

The key building blocks of the combined feedback-feedforward controller are developed next.

\subsubsection{Internal Model}

Let $\left(\boldsymbol{A}_{I}, \boldsymbol{B}_{I}, \boldsymbol{C}_{I}\right)$ be the internal model of the reference waveform $q_{i d}(k)$ and the deterministic disturbance $d_{d d i}(k)$ :

$$
\boldsymbol{x}_{I}(k+1)=\boldsymbol{A}_{I} \boldsymbol{x}_{I}(k)+\boldsymbol{B}_{I} u_{I}(k)
$$

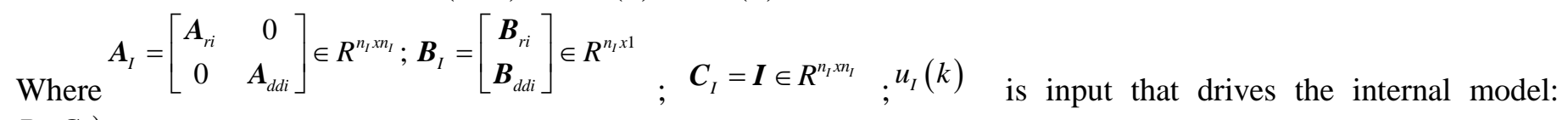
$\left(\boldsymbol{A}_{I}, \boldsymbol{B}_{I}, \boldsymbol{C}_{I}\right)$ generates the class of all reference and disturbance waveforms

\subsubsection{Feedback Controller}

The state-feedback strategy is obtained using the separation principle governing the estimation of the states, and the implementation of state-feedback strategy. First, the state-feedback strategy is derived using the linear quadratic optimization approach assuming that the states of the signal model are all available. Then the states of the signal model are substituted by those estimated by the associated KF [14]. The state feedback law is:

\subsubsection{Feed-Forward Controller}

$$
u_{f b i}(k)=-\boldsymbol{F}_{p} \boldsymbol{x}_{p}(k)-\boldsymbol{F}_{I} \boldsymbol{x}_{I}(k)
$$

Even in the presence of model perturbations, the feedforward controller can mitigate the effect of the output error on the performance of the combined controller. The feedforward controller rejects quickly the output error without waiting for the deviation in the output to occur, hence its anticipatory action. The output error and the reference input are both injected into the feedforward path to reject the effects of the output error on the output, thereby ensuring that the output is generated by the reference input alone, i.e. not by the output error. The feedforward controller is given by:

$$
H_{i d} q_{d i}(z) G_{p}(z)=1
$$

Where $H_{i d}$ is the inverse of the signal model $\left(\boldsymbol{A}_{p}-\boldsymbol{B}_{p} F_{p}, \boldsymbol{B}_{p}, \boldsymbol{C}_{p}\right)$ at the frequencies of the reference signal $q_{d i}(z)$

\subsubsection{Closed-Loop System}

Substituting the feedback $u_{i f b}(k)$ and feedforward control strategies ${ }_{f f i}(k)$ given in (19), and ${ }^{u_{f f i}}(k)$ (20), (17) becomes:

$$
u_{i}(k)=-\boldsymbol{F}_{p} \boldsymbol{x}_{p}(k)-\boldsymbol{F}_{I} \boldsymbol{x}_{I}(k)+H_{r i} q_{d i}(k)
$$

Substituting $u_{i}(k)$ in the open-loop model (15) yields: 


$$
\begin{aligned}
& {\left[\begin{array}{c}
\boldsymbol{x}_{p}(k+1) \\
\boldsymbol{x}_{I}(k+1)
\end{array}\right]=\left[\begin{array}{cc}
\boldsymbol{A}_{p}-\boldsymbol{B}_{p} \boldsymbol{F}_{p} & -\boldsymbol{B}_{p} \boldsymbol{F}_{I} \\
-\boldsymbol{B}_{I} \boldsymbol{C}_{p} & \boldsymbol{A}_{I}
\end{array}\right]\left[\begin{array}{c}
\boldsymbol{x}_{p}(k) \\
\boldsymbol{x}_{I}(k)
\end{array}\right]+\left[\begin{array}{c}
\boldsymbol{B}_{p} \\
\boldsymbol{B}_{I}
\end{array}\right] q_{d i}(k)-\left[\begin{array}{c}
0 \\
\boldsymbol{B}_{I}
\end{array}\right] \vartheta_{r d i}(k)} \\
& y_{i}^{c l}(k)=\boldsymbol{C}_{p} \boldsymbol{x}_{p}(k)+\vartheta_{i}
\end{aligned}
$$

Fig. 1 shows the block diagram representation of the closed-loop system.

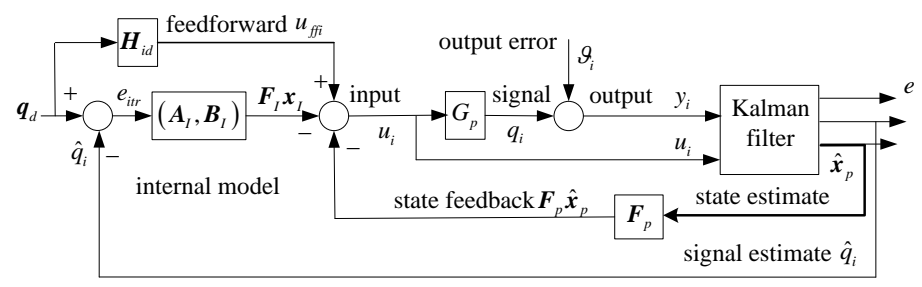

Fig. 1: Block diagram representation of the closed-loop system

Thanks to the availability of the estimates of the KF of the signal model states $\hat{\boldsymbol{x}}_{p}(k)$, the signal $q_{i}(k)$ as well as the output error $\vartheta_{i}(k)$, a novel combined feedback-feedforward controller is implemented.

The proposed controller scheme, including its tracking control performance, was evaluated on a physical two-link robot manipulator whose block diagram is shown in Fig. 1. A two-link robot manipulator is a basic classical and simple example of a robot for the evaluation of the modeling, identification and control tasks. It acts like human arms.

\section{Kalman Filter-Based Combined Controller: An Illustrative Example.}

\subsection{System identification: Three important Lemmas}

The KF residual model forms the backbone of the proposed system identification scheme that is at the core of the proposed combined controller. The KF plays a vital role in the identification tasks, including the estimation of the joint variable $^{q_{i}}$ from the corrupted output ${ }_{i}$, and in the implementation of the controller to ensure an accurate tracking of the desired trajectory. The system identification hinges on 3 important new Lemmas, whose details and proofs can be found in [12-16]. Lemma 1 sets out the conditions for the identification of the signal and signal model. Lemma 2 deals with the estimation of the signal model $\hat{G}_{p}(z)$, signal $\hat{q}_{i}(z)$ Error! Reference source not found., and output error (14), and finally Lemma provides the whitening property by showing that the KF whitens the output error (14).

\subsection{Illustrative example}

The proposed combined state-feedback and feedforward controller was evaluated on the on the physical robot manipulator. The control objective is for the end effector $\boldsymbol{p}_{d}=\left(x_{d}, y_{d}\right) \in R^{2}$, where $x_{d}(k)=a_{1}+a_{2} \cos \left(\theta_{d}(k)\right)$, $y_{d}(k)=a_{2} \sin \left(\theta_{d}(k)\right)$ so that the end-effector traces a circular trajectory in the $(x, y)$-plane. Using the inverse kinematic equation,(5), input trajectory namely the roll $\phi_{d}$, and pitch $\theta_{d}$ and thrust $f_{\text {thd }}$ estimated by the inverse kinematics equation so that it tracks the desired translation trajectory $\left(x_{d}, y_{d}, z_{d}\right)$. The internal model $\left(\boldsymbol{A}_{I}, \boldsymbol{B}_{I}, \boldsymbol{C}_{I}\right)$ is:

$$
\boldsymbol{x}_{I}(k+1)=\left[\begin{array}{cc}
2 \cos \omega & -1 \\
1 & 0
\end{array}\right] \boldsymbol{x}_{I}(k)+\left[\begin{array}{l}
1 \\
0
\end{array}\right] e_{t r}(k)
$$

The stochastic disturbance Error! Reference source not found.

$$
\begin{aligned}
& \boldsymbol{x}_{d r i}(k)=\left[\begin{array}{cc}
0.3960 & -0.8025 \\
1 & 0
\end{array}\right] \boldsymbol{x}_{d r i}(k)+\left[\begin{array}{l}
1 \\
0
\end{array}\right] w_{i}(k) \\
& d_{r i}(k)=\left[\begin{array}{ll}
1.4160 & 0
\end{array}\right] \boldsymbol{x}_{d r i}(k)
\end{aligned}
$$


Fig. 2 shows the signal $s_{i}^{c l}(k)$ and the output $y_{i}^{c l}(k)$ of the closed-loop system. Subfigures A and B show the corrupted outputs while $\mathrm{C}$ and $\mathrm{D}$ shows the signal outputs and the true signals.

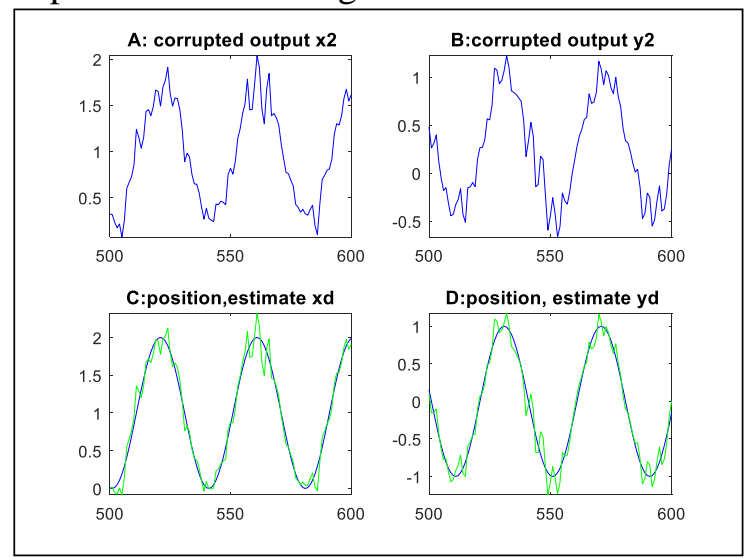

Fig. 2 Outputs using proposed internal model-based controller

\section{Kalman Filter-Based Estimation of the Signal and its Derivatives}

The dynamic model of the robot model is a function of the joint variable, namely the signal $\boldsymbol{q}$, its first derivative $\dot{\boldsymbol{q}}$ and its second derivative $\ddot{q}$. The joint variables ad their derivatives need to be estimated from the output corrupted by disturbances and measurement noise. A new KF-based derivative-free soft sensor proposed in [17] for accurate estimation replacing the maintenance-prone and noise-corrupted hardware sensors is generalized here to a wider class of robotic systems when the output is corrupted by stochastic and deterministic signals and measurement noise. The model of the soft sensor and the associated KF are identified directly without any a-prior knowledge or use of the covariance of the disturbance and measurement noise The KF estimates using the key properties of the signals, and their derivatives $\hat{\theta}_{1}, \hat{\theta}_{1}, \hat{\theta}_{1}, \hat{\theta}_{2}$, $\hat{\theta}_{2}$ and $\hat{\ddot{\theta}}_{2}$ from the output of the joint angles buried in the stochastic disturbance and measurement noise are accurate, thanks in great part to the use of the data-enriching emulators.

\section{Conclusions}

In this paper, a new internal model for tracking the desired trajectory, and the KF-, based combined feedforwardfeedback controller was proposed, and developed for a wider class of systems governed by the general Box-Jenkins model. The KF estimates accurately the pertinent signals and their derivatives from the output corrupted by deterministic and stochastic disturbance and measurement noise. The tracking error is selected as the error between the reference trajectory and the KF estimate of the signal, rather than the noisy output. Further, the state-feedback law was implemented using the accurate and reliable noise-free estimates of the states of the identified KF. As a result, the trajectory of the end-effect was accurate and did not exhibit any random deviations about the reference trajectory. As a by-product of this study, a soft sensor using derivative-free Kalman filtering was developed and used to estimate the signal and their derivatives, replacing thereby the maintenance and noise-prone hardware sensor. The proposed controller was successfully evaluated both by simulation and on a physical robot and shown to be significantly superior to the conventional controller, and its tracking error was also shown to be negligibly small. This gives ample encouragement to expand the proposed design to several other important application areas, including industrial and medical ones.

\section{Acknowledgements}

The authors acknowledge the support of Professor Marcelo Ramirez of the Universidad Mayor de San Andres, La Paz Bolivia in evaluating the proposed scheme on a physical robot. and the graduated students Eduardo Jair Tito Mamani; Paulo 
Roberto Torrico, Edmundo Cayo Morales Arinez, Mariano Bianco Lino Sanchez, Justo Franz Choque Choque, Bryan Saravia Quisbert, Edgar Gonzales Laura, Eduardo Laura Pizarro for their help in modeling, identification, control, implementation and evaluation of the proposed scheme on a physical Loma Marconi; Pamala Giselle Villarroel, Paul Gerardo Cori Mamani, Claudio Cesar Carlos Olivares; Laydi Aydee Cruz robot.

\section{References}

[1] F. L. Lewis, Darren M. Dawson, and C.T. Abdallah (2003), "Robot Manipulator Control: Theory and Practice", CRC Press; Published December 12, 2003 ISBN 9780824740726; Automation and Control Engineering, Marcel Dekker, Inc., New York.

[2] M. Ben-Ari and F.Mondada (2018), "Elements of Robotics", Springer Open, ISBN 978-3-319-62532-4

[3] R. Taylor,( 2004), "Medical robotics and computer integrated surgery", NSF Workshop on status of robotics in the United States, Arlington, Virginia, July 21-22.

[4] M. W. Spong, Seth Hutchinson, M. Vidyasagar (2006), "Robot Modeling and Control” John Wiley \& Sons, New York, ISBN 04716499029780471649908

[5] J. Shah, S.S. Rattan, B.C. Nakra, "Dynamic Analysis of Two Link Robot Manipulator for Control Design Using Computed Torque Control”, International Journal of Research in Computer Applications and Robotics, ISSN 23207345,vol.3, issue, Pg. 52-59, January 2015

[6] X.Yuan, B. Huang, Y.Wang, C. Yang, Weihua Gu , (2018), "Deep Learning-Based Feature Representation and Its Application for Soft Sensor Modeling With Variable-Wise Weighted SAE" IEEE Transactions on Industrial Informatics Vol. 14, No. 7,

[7] D. Abbott (2014), "Applied Predictive Analytics: Principles and Techniques for the Professional Data Analyst", John Wiley \& Sons,

[8] G. J. Mallory and R. Doraiswami (1999), “A Filter for On-line Estimation of Spectral Content”, IEEE Transactions on Instrumentation and Measurement, Vol. 48, No.6, pp. 1047-1055

[9] P. K. Khosla, T. Kaneda, Experimental of Evaluation of Nonlinear Feedback and Feedforward Control Schemes for Manipulators, The International Journal of Robotics Research, Vol. 7, No. 1, February 1988.

[10] J.J.E. Slotine, W. Li, Adaptive Manipulator Control: A Case Study, IEEE Trans. Automat. Control 33, 1988, 9951003

[11] Huiming Wang, Yongping Pan, Shihua Li and Haoyong Yu ,"Robust Sliding Mode Control for Robots Driven by Compliant Actuators" IEEE Transactions on Control Systems Technology, Vol. 27, No. 3, May 2019

[12] M. Lu, L. Liu , G Feng, "Adaptive tracking control of uncertain Euler-Lagrange systems subject to external disturbances" Automatica, 104 (2019) 207-219

[13] J.Peng, and Y. Liu, “Adaptive Robust Quadratic Stabilization Tracking Control for Robotic System with Uncertainties and External Disturbances", Journal of Control Science and Engineering DOI: 10.1155/2014/715250 Sep 16, 2014

[14] W. He, W.Ge, Y. Li, Y-J Liu, C. Yang and C. Sun," Model Identification and Control Design for a Humanoid Robot”, IEEE Transactions on Systems, Man, and Cybernetics: Vol. 47, No. 1, January 201745

[15] R., Doraiswami and L. Cheded (2017), Robust Fault-Tolerant Control Using an Accurate Emulator-Based Identification Technique, International Journal of Control, ISSN: 0020-7179, 1336-5820 (on line) Journal homepage: http://www.tandfonline.com/loi/tcon20

[16] R. Doraiswami, and L Cheded (2018), Robust Kalman filter-based least squares identification of a multivariable system, IET Control Theory and Applications, ISSN 1751-8644 doi: 10.1049/iet-cta.2017.0829, www.ietdl.org, pp.1-11

[17] R. Doraiswami, L. Cheded, "Novel direct and accurate identification of Kalman filter for general systems described by a Box- Jenkins model" Invited Book Chapter "Kalman Filter" (ISBN: Edited by Dr. Felix Govaers and published by InTech, May 2019.

[18] B. Francis, and M. W. Wonham. (1976). "The Internal Model Principle of Control Theory." Automatica 12: 457-465

[19] B.G. Rigados, (2012), "Derivative-free Kalman Filtering for sensor-less control of MIMO nonlinear dynamical systems", Proceedings of 2012 IEEE International Conference on Mechatronics and Automation 